\title{
Florid Reactive Periostitis of the Hand: Three Case Report and Review of the Literature
}

\section{María Jesús Rivera Vegas*}

Plastic, Aesthetic and Reconstructive Surgery. Hospital Universitario de Burgos, Spain

*Corresponding Author: María Jesús Rivera Vegas, Plastic, Aesthetic and Reconstructive Surgery. Hospital Universitario de Burgos, Spain.

Received: July 29, 2019; Published: August 19, 2019

\begin{abstract}
Reactive florid periostitis is a benign reactive lesion relatively rare The lesion usually occurs in the finger and seldom in the metacarpal. The importance of knowing the florid reactive periostitis is that it can lead to diagnostic errors with more serious lesions such as infections and malignant bone tumors, avoiding aggressive surgeries. We present three clinical cases, one in the proximal phalanx, another in the metacarpal, which is an unusual location and the other is located in the hypothenar eminence. Reactive florid periostitis should be adequately diagnosed by biopsy.

Keywords: Florid Reactive Periostitis; Osseous Tumor; Hand; Metacarpals; Phalanges
\end{abstract}

\section{Abbreviations}

CT: Computed Tomography; MRI: Magnetic Resonance Imaging

\section{Introduction}

Reactive florid periostitis is a benign reactive lesion relatively rare that can be confused clinically and radiologically with osteosarcoma and osteomyelitis.

There is a great variety of names to refer to this entity as parosteal fasciitis [1], fasciitis ossificans [2], benign fibro-osseous pseudotumor [3], pseudomalignant osseous tumor of soft tissue and nodular fasciitis $[4,5]$.

We present 3 clinical cases, one of them in proximal phalanx, other in hypothenar eminence and another in the interosseous space between $2^{\text {nd }}$ and $3^{\text {rd }}$ metacarpal.

\section{Clinical Cases}

Case No. 1

A 61-year-old patient presented a lesion at the level of the proximal phalanx of the third finger of the right hand of months of evolution, of slow growth.

Her personal history: diabetes mellitus type II in habitual treatment with metformin.

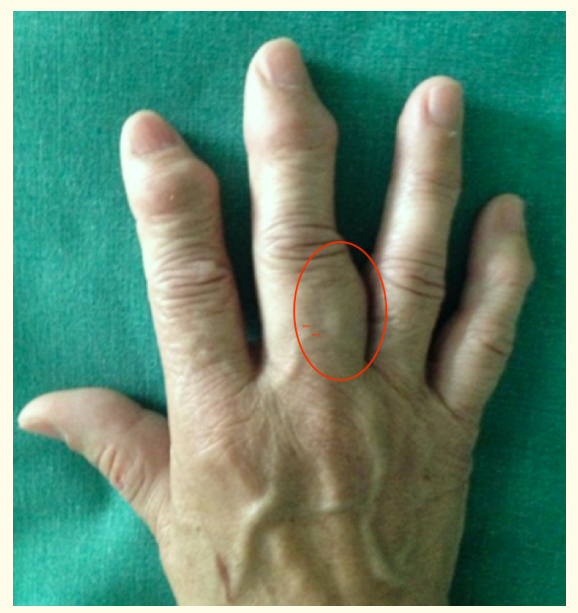

Figure 1: Observe an elevated area on the ulnar edge of the proximal phalanx of the third finger of the right hand.

It is a hard lesion attached to the deep plane.

On examination, the patient presented Heberden nodules on both hands. At the level of the ulnar border of the $3^{\text {rd }}$ finger on the right hand, a hard lesion adheres to the deep plane, not mobile or painful on palpation. (Figure 1).

In simple radiology, there are marked degenerative changes in distal interphalangeal and rhizarthrosis of both thumbs. In the proximal phalanx of the $3^{\text {rd }}$ finger of the right hand there is bulging 
of soft parts in the ulnar diaphysis (Figure 2) that compared to a $\mathrm{x}$-ray done two months after, there was an increase in the calcification of the lesion as well as an increase of the reinforcement of the adjacent cortex (Figure 3).

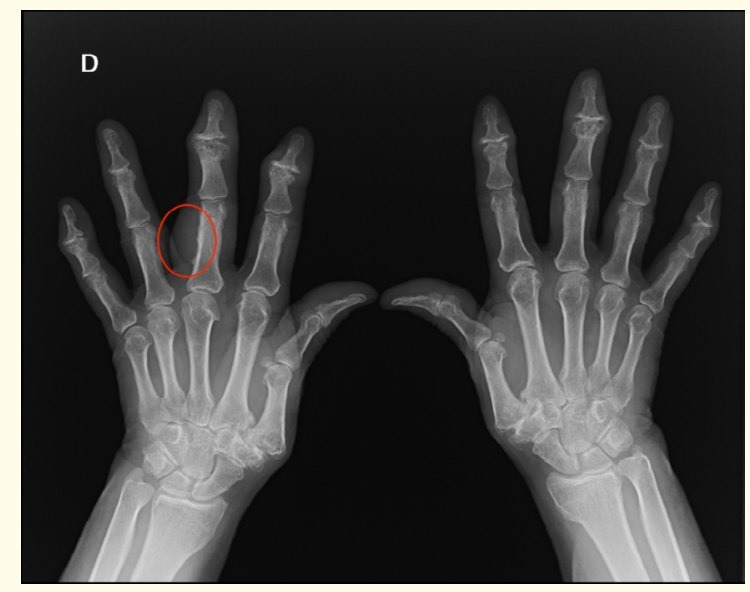

Figure 2: This is an x-ray taken 2 months before being seen in the plastic surgery office. We can see a beginning of the injury with a soft tissue involvement.

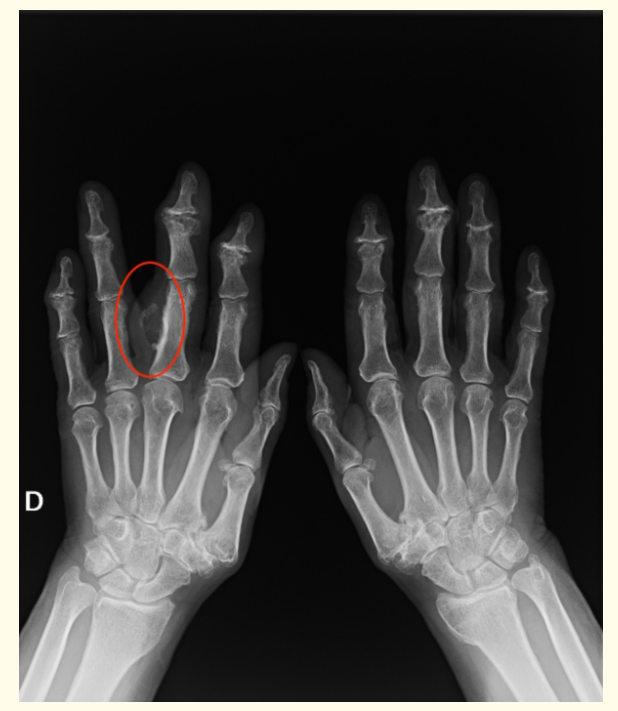

Figure 3: In X-ray, there was soft tissue protuberances in the proximal phalanx of the 3rd finger of the right hand that correspond to perioseal calcifications. The calcifications have grown during the two months between both radiographs.

In CT, a soft tissue lesion is identified, which causes discrete scalloping of the bone cortex of the ulnar margin of the diaphysis and distal metaphysis of the proximal third finger phalanx. This lesion seems to show a partial periosteal and discrete calcified matrix, with approximate diameters of $12 \times 8 \times 17 \mathrm{~mm}$. These findings, due to their characteristics and location, follow the following dif- ferential diagnoses as the first diagnostic possibilities: periosteum chondrosarcoma, low-grade periosteum chondrosarcoma, periosteal osteosarcoma. (Figure 4) The patient underwent surgery to remove the lesion (Figure 5) with anatomopathological diagnosis of fibrous reactive periostitis.

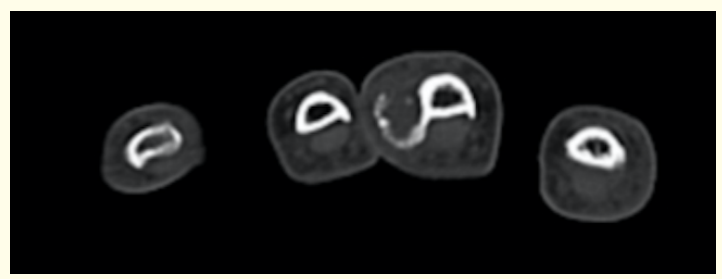

Figure 4: On axial image CT, This lesion seems to show a partial and discrete periodic calcified matrix, with approximate diameters of $12 \times 8 \times 17 \mathrm{~mm}$. and it suggests the following differential diagnoses such as the first diagnostic possibilities: periosteal chondrosarcoma, low grade periosteal chondrosarcoma, periosteal osteosarcoma.

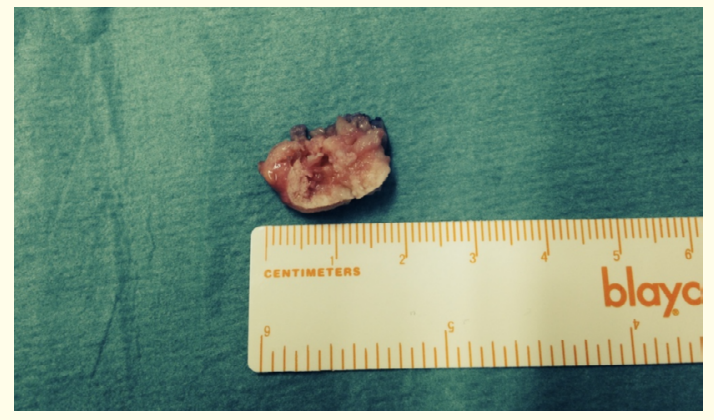

Figure 5: Perioperative image after tumor excision.

After 4 years of postoperative follow-up, the patient is free of disease (Figure 6).

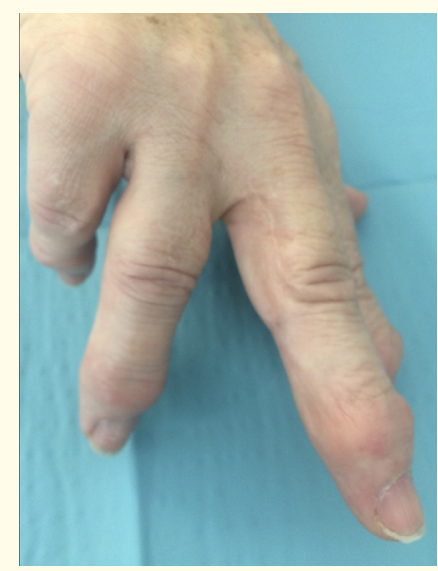

Figure 6: Four years after surgery, no clinical signs or radiological recurrence of injury. 
Case No. 2

A 55-year-old patient with no personal history of interest except generalized arthrosis. She was attendant in outpatient plastic surgery because she had a radial tumor on the radial border of the head / neck of the 3rd metacarpal right hand. On examination, the lesion is painful to pressure and movements with this simple radiology (Figure 7).

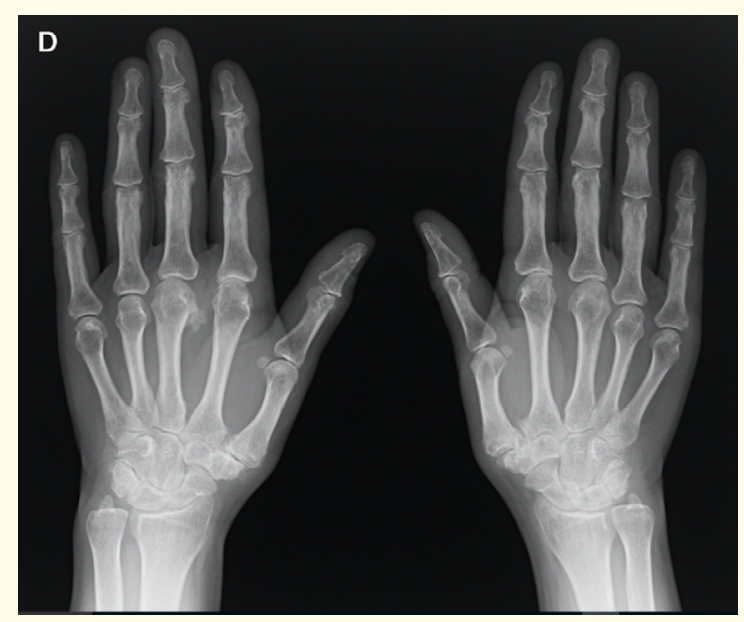

Figure 7: In simple radiology, the patient presented a calcification of spiculated form In the second interosseous space in right hand.

The patient underwent MRI of the right hand, at the height of the dorsal interosseous space between the heads of the $2^{\text {nd }}$ and 3 rd metacarpal, a nodular, ovoid lesion of $17 \times 15 \times 10 \mathrm{~mm}$ of subcutaneous location was observed that seems to correspond to mature bone with a bone nucleus. or calcified surrounded by a peripheral shell of tissue other than bone, with a chondral aspect, with possible contact of this lesion with the joint capsule and with the periosteum of the 3rd metacarpal, suggesting the possibility of a Nora lesion with differential diagnosis with a fibroosseous pseudotumor, chondroma or periosteal osteochondroma, ossifying myositis and with less possibility of paraostal osteosarcoma (Figure 8).

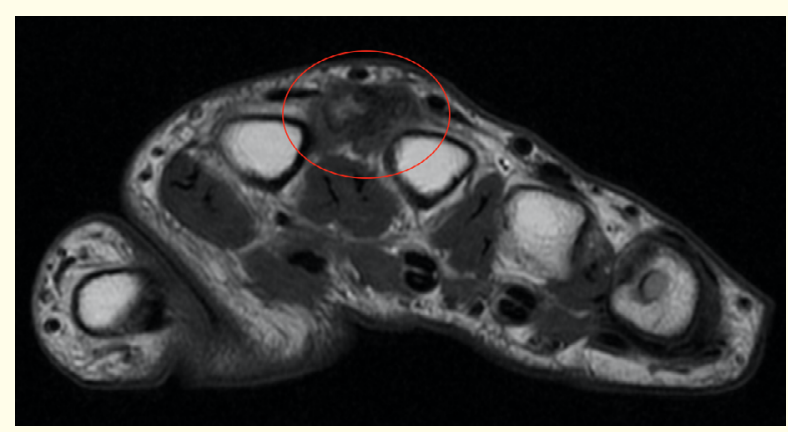

Figure 8: In axial RMI, the patient presented calcified subcutaneous nodule that may suggest Nora's lesion, fibrous pseudotumor, chondroma, ossifying myositis or parostal osteosarcoma.
The TC made not provide more information (Figure 9).

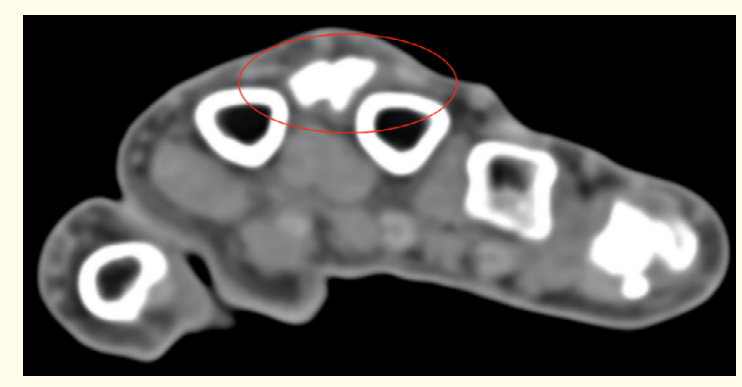

Figure 9: TC, axial image of the same lesion.

The lesion was extirpated and in the anatomopathological study it was diagnosed as reactive florid periostitis.

After two years of follow-up, the patient did not show signs of recurrence of the lesion (Figure10).

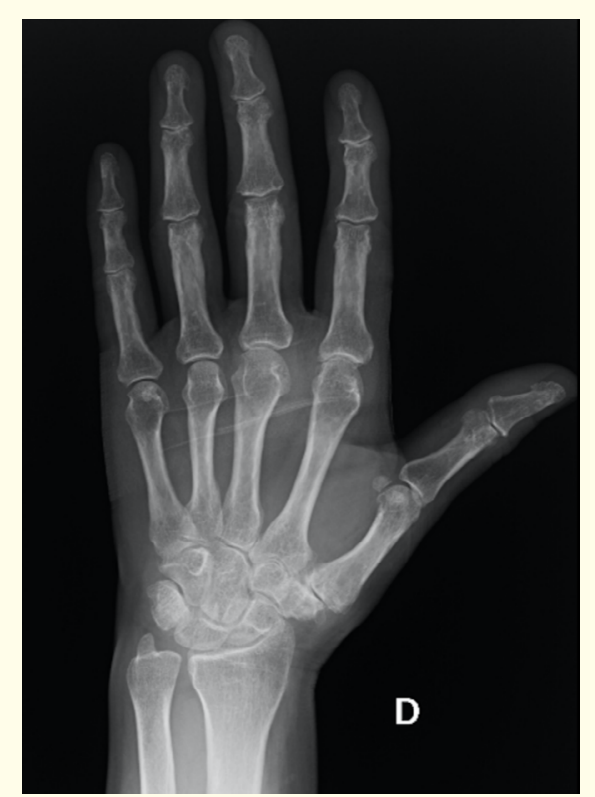

Figure 10: After two years there are no signs of recurrence of the lesion.

Case No. 3

A 39-year-old patient, with no personal history of interest, went to the outpatient Plastic Surgery, she presented a hard mass of months of evolution in right-hand hypothenar eminence.

The MRI, a solid mass of superficial soft parts with a local infiltrative component, an irregular contour spiculated at the level of the 5th metacarpal. (Figure 11) (Figure 12) Since the clinical or radiological presentation was not the usual one, we sent the patho- 
logical anatomy piece to several hospitals to confirm the diagnosis of reactive fibrous periostitis.

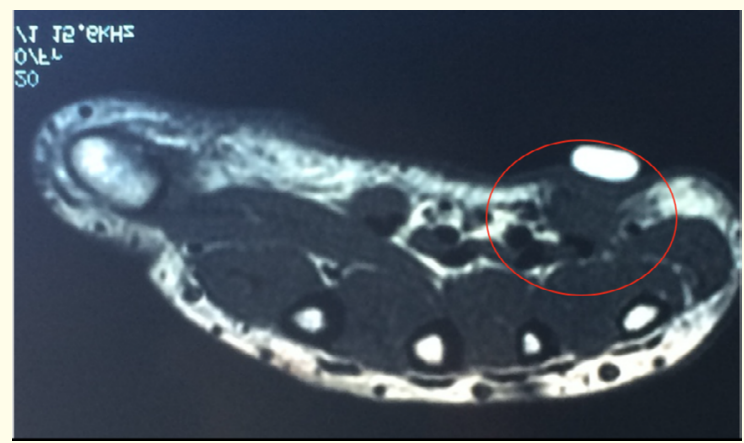

Figure 11: Axial RMI solid tumor in the palmar region of the right hand at the level of the fifth metacarpal with irregular spiculated contour of difficult radiological assessment.

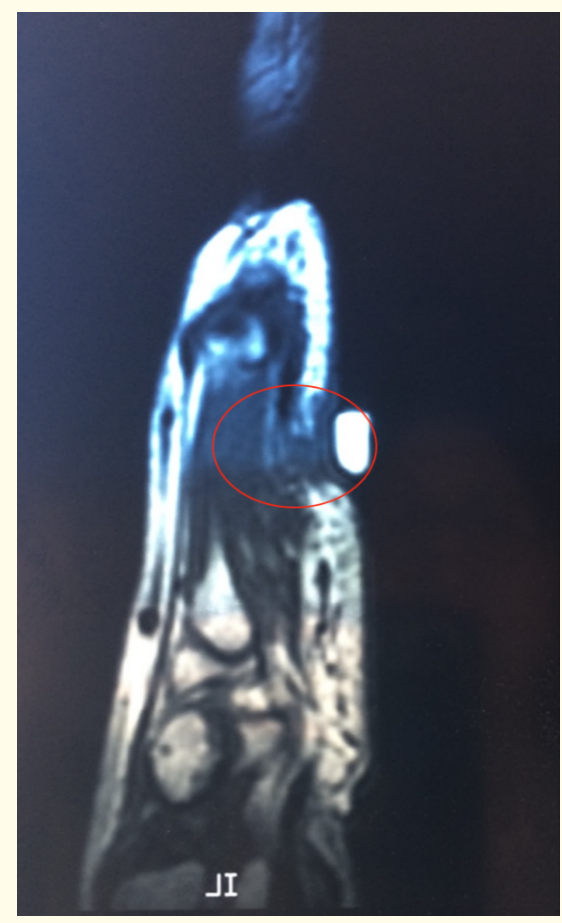

Figure 12: Sagittal RMI of the same imagen.

\section{Discussion}

Reactive fibrous periostitis is a relatively rare and poorly understood lesion.

In 1933, Mallory presented 4 tumors that are characterized by an increase in soft tissue containing cartilage and bone [6]. The name reactive fibrous periostitis is due to Spjut and Dorfman in 1981, authors who presented the first long series, and changed the name of the pathological entity of nodular fasciitis or ossifying fasciitis to reactive fibrous periostitis, due to its relationship with the periosteum and not with the fascia [7]. Dupree., et al. presented the longest series formed by 21 patients, and described it as a subcutaneous variant of ossifying myositis, called pseudotumor fibroosseus. Radiologically they observed that the tumors are adjacent although separated from the bone and these authors do not believe that the periosteum is their main site of origin [3].

The etiology of reactive fibrous periostitis is not known. There has been talk of repeated microtrauma as an etiological possibility, although the previous history of trauma is collected in less than $50 \%$ of patients [8]. Benign ossifying lesions can occur in the dermis, subcutaneous cellular tissue, tendons, fascia, muscles and periosteum. When they occur in relation to the periosteum, they form a group of lesions that are called reactive lesions of the bone surface where we find reactive florid periostitis, osteochondromatose bizarre periosteal proliferation and osteochondroma, which, according to some authors, represent different phases of a reactive process. Yuen., et al. [9] propose a unitary hypothesis due to the overlap of these nosological entities. These authors consider the existence of an initial stimulus often related to a trauma that causes a subperiosteal hemorrhage, although it is not reported by the patient in up to $50 \%$ of cases. If the reaction is maintained in the periosteum, a fusiform periostitis develops with the cortex intact, the fusiform aspect is due to the vascularization of the subperiosteal plexus. The focus of mature ossification and this periostitis is incorporated into the cortex, combining with a widening of the vascular periosteum. These biopsied lesions would give a pathological anatomy of reactive fibrous periostitis with fibroblasts and minimal osteocartilaginous proliferation. If the periosteum ruptures, the reactive process extends into the tissue that surrounds it and transforms a fusiform process into a lobular process, with a pathological diagnosis of periosteal bizarre osteochondromatose proliferation, with bone and metaplastic cartilage. The periostal bizarre osteochondromatose proliferation was described by Nora in 1983, [10] also known by this name, Nora's Lesion. Finally, the focus of ossification matures with the formation of bone in the base that is incorporated into the underlying bone and in the periphery islands of cartilage (osteochondroma) persist due to the loss of complete ossification since the vascularization of the process is carried out through the surrounding tissue that is the practically avascular fat tissue.

According to this hypothesis, the local anatomy can modify the process, so in the volar face of the distal phalanx of the fingers, the subcutaneous presents strong septa that would prevent the expansion of the lesions beyond the periosteum, hence the difficulty of finding lesions in the distal phalanges [11]. 
Sundaram., et al. [12] are the only authors that confirm the clinical progression of reactive fibrous periostitis to osteochondromatose bizarre periosteal proliferation in a young patient, 11 years old, in which after ruling out an infectious or malignant tumor process and performing a biopsy with diagnosis of periostitis fibrous reactive, surgery was delayed for 6 months with the patient being monitored in outpatient visits by applying the same criteria used in the presentation of these lesions in the long bones in order to facilitate their exeresis and observed a modification of the images radiographs during that period of time, towards a periosteal osteochondromatose proliferation that was confirmed with the pathological anatomy. These authors conclude, based on their experience, a possible temporary progression of reactive fibrous periostitis to osteochondromatose bizarre periosteal proliferation in some cases in others fibrous reactive periostitis remains a selflimiting process.

The symptoms are similar in any age group, being more frequent its appearance in the second or third decade of life although the age variations in the presented series are very wide, from 5 to 70 years. It is more frequent the presentation in women, coinciding with our clinical cases $[13,14]$. The main clinical finding is the existence of a mass, which sometimes coexists with sensation of pressure and erythema. In the case of reactive fibrous periostitis with integrity of the periosteum, the occurrence of pain due to the traction exerted on the nerve endings of the intact periosteum is frequent, but not in the case of osteochondromatose bizarre periosteal proliferation where the periosteum is already broken, the pain disappearing. In $60 \%$ of reactive fibrous periostitis is located in the proximal phalanx $[15,16]$ the rest of the locations in the phalanges and metacarpals are rare $[17,18]$. The long fingers are affected in the same proportion except the thumb, in which its appearance is rare $[19,20]$.

Radiographically, reactive fibrous periostitis is characterized by soft tissue inflammation with a variable periosteal reaction, with juxtacortical calcification occurring in the soft tissues with the underlying cortex intact [21]. Cortical continuity may help to differentiate this process from other lesions with a less favorable prognosis such as periosteal chondrosarcoma or periosteal osteochondroma [22]. Occasionally the cortical lesion can be observed although rare, and in the cases in which it was presented, it was not associated with a negative evolution [23].

The diagnosis must be confirmed with the biopsy of the lesion [13-16,24].

Other benign lesions among which differential diagnosis will be ossifying myositis in mature state, but this is not related to the periosteum and histologically presents what is known as "zone effect" characterized by a middle zone containing osteoblasts with mature bone island and in the periphery there is mature trabecula, there is a centrifugal maturation, this pathological anatomy does not exist in reactive fibrous periostitis. This lesion is characterized radiographically by a paraphyseal mass that contains radiodense images particularly in the periphery and with the passage of time ends as mature bone separated from the adjacent periosteum by a radiolucent area [25].

Osteomyelitis also presents a periosteal proliferation with an anatomopathological diagnosis of inflammatory cell infiltrate that does not appear in reactive florid periostitis. Subacute osteomyelitis due to Pasteurela is very representative and has been described as a cause of monototic periosteal in the bones of the hands $[16,17,22]$.

In the case of malignant lesion, we must consider periosteal osteochondrosarcoma and periosteal osteosarcoma, parostal osteosarcoma characterized by destruction of the periosteum, cortical, even spongy and present in the anatomopathological study atypical mitotic figures and osteoblastic pleomorphism in the case of periostal osteosarcoma or cartilaginous anaplasic cells in the case of osteochondrosarcoma[23].

The evolution of reactive fibrous periostitis is benign and there have even been cases in which they have resolved spontaneously [26].

The proposed treatment would be a simple excision, leaving the amputation of the radius for the cases of recurrences, surgical attitude maintained by most of the authors.

Patel [27] recommends applying Enneking's system for the surgical staging of benign tumors in the case of reactive fibrous periostitis to assess the different tumor recurrences. These investigators concluded that local excision would be appropriate for less aggressive tumors in stage I, but would not be sufficient for grades II and III, lesions that would require a radio amputation to prevent recurrence.

\section{Conclusion}

The importance of knowing the florid reactive periostitis is that it can lead to diagnostic errors with more serious lesions such as infections and malignant bone tumors, avoiding aggressive surgeries.

When we find bone tumors in the hand suspected of malignancy or compatible with infection that does not progress well with treat- 
ment, we would consider florid reactive periostitis as a differential diagnosis.

\section{Conflict of Interest}

Declare if any financial interest or any conflict of interest exists.

\section{Bibliography}

1. Hutter RVP., et al. "Parosteal fasciitis: a self-limited benign process that simulates a malignant neoplasm". The American Journal of Surgery 104 (1962): 800-807.

2. Kwittken J and Branche M. "Fasciitis ossificans". American Journal of Clinical Pathology 51 (1969): 251-255.

3. Dupree WB and Enzinger FM. "Fibro-osseous pseudotumor of the digits". Cancer 58 (1986): 2103-2109.

4. McCarthy EF, et al. "Parosteal (nodular) fasciitis of the hand: a case report". Journal of Bone and Joint Surgery 58A (1976): 714-716.

5. Price EB Jr., et al. "Nodular fasciitis: a clinicopathologic analysis of 65 cases". American Journal of Clinical Pathology 35 (1961): 122-136.

6. Mallory TB. "A group of metaplastic and neoplastic bone and cartilage containing tumors of soft parts". The American Journal of Pathology 9 (1933): 765-776.

7. Spjut HJ and Dorfman HD. "Florid reactive periostitis of the tubular bones of the hands and feet: a benign lesion which may simulate osteosarcoma". The American Journal of Surgical Pathology 5 (1981): 423-433.

8. Tomori Y., et al. "Florid reactive periostitis in the fifth phalange of a professional boxer". Medicine 95 (2016): 51-54.

9. Yuen M., et al. "Proliferative periosteal processes of phalanges: a unitary hypothesis". Skeletal Radiology 21 (1992): 301303.

10. Nora FE., et al. "Bizarre parosteal osteochondromatous proliferations of hand and feet". The American Journal of Surgical Pathology 7 (1983): 245-250.

11. Landsman JC., et al. "Florid reactive periostitis of the digits". Orthopedic Reviews 19 1990: 828- 834.

12. Sundaram M., et al. "Florid reactive periostitis and bizarre parosteal osteochondromatous proliferation: pre- biopsy imaging evolution, treatment and outcome". Skeletal Radiology 30 (2001): 192-198

13. Kandzierski G and Matuszewski L. "Florid-reactive Periostitis of the Phalanges: A 2-Case Report". Journal of Pediatric Orthopaedics 32 (2012): 179-183.
14. Vinglas R and Schnall SB. "Florid Reactive Periostitis of the Hand". The American Journal of Orthopedics39 (8) (2010): 396-398.

15. Craver RD., et al. "Florid reactive periostitis of the Hand". Human Pathology 28 (1997): 745-747.

16. Holmes WS., et al. "Case report 413: Florid reactive periostitis of the proximal phalanx of the fourth finger (parosteal fasciitis, pseudosarcomatous fibromatosis, fasciitis ossificans)". Skeletal Radiology 16.2 (1987): 163-165.

17. Gao Z., et al. "Florid Reactive Periostitis of the Metacarpal and Phalanx: 2 Case Reports". Journal of Hand Surgery 38A (2013): $2134 \mathrm{e} 2137$.

18. Rogers GF and Brzezienski MA. "Florid Reactive Periostitis of the Middle Phalanx: A Case Report and Review of the Literature". Journal of Hand Surgery 24 A (1999): 1014-1018.

19. Solana J., et al. "Florid reactive periostitis of the thumb: a case report and review of the literature". Chirurgie de la Main 22 (2) (2003): 99e103.

20. Riaz M., et al. "Florid reactive periostitis of the thumb". The Journal of Hand Surgery: British 21.2 (1996): 276-279.

21. Porter AR., et al. "Florid Reactive Periostitis of the Phalanges". American Journal of Roentgenology 144 (1985): 617-618.

22. Jambhekar NA., et al. "Florid reactive periostitis of the hands". Skeletal Radiology 33 (2004): 663-665.

23. Howard RF., et al. "Florid reactive periostitis of the digit with cortical erosion: A case report and review of the literature". Journal of Hand Surgery 21 A (1996): 501-505.

24. Kovach JC., et al. "Florid reactive periostitis". Journal of Hand Surgery 11A (1986): 902-905.

25. Al-Qattan MM., et al. "Manegement of Myositis ossificans of the hand: A case Report and a Review of the Literature". Journal of Hand Surgery American2017: 576.e1-576.e4.

26. Sakamoto A., et al. "Florid reactive periostitis of the metacarpal bone: a case of resolution without resection". Journal of Hand Surgery European 43 (10) 2018: 1118-1119.

27. Patel MR and Desai SS. "Pseudomalignant osseus tumor of soft tissue: A case report and review of the literature". Journal of Hand Surgery American 11.1 (1986): 66-70.

Volume 2 Issue 9 September 2019 (C) All rights are reserved by María Jesús Rivera Vegas. 